\title{
The GALS locomotor screen and disability
}

\author{
M J Plant, S Linton, E Dodd, P W Jones, P T Dawes
}

\begin{abstract}
Objectives-Examination of the locomotor system is frequently neglected. Therefore, the GALS locomotor screen (Gait, Arms, Legs, Spine) has been proposed by Doherty et al as a practical method of identifying functionally important problems. This study was designed to test whether this screen reflects functional impairment, as measured by accepted health status measures.

Methods-Two observers performed the GALS screen in a total of 83 patients with a variety of musculoskeletal conditions. The examination components of GALS were rated by a simple 0 to 3 scale. Physical ability was further assessed by Health Activity Questionnaire (HAQ), Barthel index and Steinbrocker's ARA classification.
\end{abstract}

Results-For the total patient group, Spearman correlations between GALS and the three functional indices were good $(r=0.62$ to $0.71, p<0.001)$. Correlations were equally good for rheumatoid arthritis patients alone $(r=0.65$ to 0.70 , $p<0.001$ ), but less good although still significant for the other miscellaneous rheumatic conditions $(r=0.31$ to 0.46 , $\mathrm{p}<0.05)$. Observed proportional agreement between the two observers for the individual scores was $>70 \%$, with a kappa statistic $k=0.49$ to $0 \cdot 74$.

Conclusions-The GALS screen is a reliable and valid measure of functional ability, compared with standard accepted indices in a variety of musculoskeletal diseases. This supports the proposal for its use as a screening test by general practitioners and medical students.

(Ann Rheum Dis 1993; 52: 886-890)

Rheumatology Centre Haywood Site, Haywood Site,
North Staffordshire Hospital, Stoke on Trent, United Kingdom Staffordshire

M J Plant

S Linton

P T Dawes

Department of Occupational Therapy, Staffordshire Rheumatology Centre E Dodd

Correspondence to: Dr Plant,

Haywood Hospital,

High Lane,

Burslem,

Stoke-on-Trent ST6 7AG,

United Kingdom

Accepted for publication 27 August 1993

Examination of the locomotor system is complicated, time consuming, and frequently neglected, ${ }^{1}$ even though musculo-skeletal disorders are common in both general and hospital practice. ${ }^{23}$ As a result, significant and remedial causes of morbidity may be overlooked.

A practical method is therefore required to alert medical practitioners to musculoskeletal problems: this could be incorporated in the systems review section of the history and in the clinical examination. Such a method should be quick and easy to perform, and should reliably identify problems of function.

Such a locomotor screen has been suggested by Doherty et al and has been given the acronym, GALS, which stands for Gait, Arms, Legs, and Spine. ${ }^{4}$ It comprises three questions about pain, and ability to dress and climb stairs, followed by examination of these four systems regarding appearance and movement. GALS was adapted from a 'minimal rheumatological screen' that was shown to be sensitive in detecting locomotor abnormality. ${ }^{5}$ The GALS screen has since been endorsed by the British Society for Rheumatology, and put forward for use in undergraduate teaching. ${ }^{4}$ Its ability to reflect disability, however, has not been tested and proven.

Various scales have been devised to assess functional ability either by self-administered questionnaires or assessments by health care professionals. The Steinbrocker ARA classification has been widely used by rheumatologists since its inception in 1949, and subsequent indices have been compared against it. ${ }^{6}$ The four broad subgroups were revised in 1991 to improve sensitivity. ${ }^{\text {? }}$

The Stanford Health Assessment Questionnaire (HAQ) is a well-documented self-report questionnaire, ${ }^{89}$ which assesses physical ability by a total of 20 questions on activities of daily living. It has been validated in many clinical trials ${ }^{10}{ }^{11}$ and shown to be a strong predictor of medical care utilisation, work disability and mortality,,$^{12}$ as well as being sensitive to change.

The Barthel score assesses independence in both neurological and musculoskeletal disorders. In contrast to the Steinbrocker and $\mathrm{HAQ}$, it includes questions on bowel and bladder control. It has proved reliable in a wide range of chronic diseases, ${ }^{14} 15$ and predicts survival, length of hospital stay and progress in stroke patients. ${ }^{16}$

This study was designed to assess how well the GALS screen reflects functional ability, as measured by these accepted methods, and to test reproducibility between observers. A simple scoring system was added to the GALS screen to facilitate comparison.
Gals locomotor screen

Each patient was examined by either a senior house officer or registrar in rheumatology, and 
30 patients were examined by both on the same day. First the patients were asked three questions: (1) Have you any pain or stiffness in your muscles, joints or back? (2) Can you dress yourself completely without any difficulty? (3) Can you walk up and down stairs without any difficulty?

The examination section is divided into 7 sections, as follows:

(1) Gait-patient walks 10 yards and turns around.

(2) Arms appearance.

(3) Arms movement - hands behind head

- arms outstretched

- pronate hands

- make a fist

- oppose thumb to each finger

- squeeze MCP joints.

(4) Legs appearance - includes callosities of soles

- test for knee effusion.

(5) Legs movement - patient flexes hip and knee

- internal rotation of hip

- squeeze MTP joints.

(6) Spine appearance - including palpation of supraspinatus muscle for fibromyalgia.

(7) Spine movement - lateral flexion of cervical spine

- bend forward and touch toes

- palpate lumbar interspinous distance.

Each of these 7 groups is scored on the following scale, giving a maximum score of 21 . $0=$ normal

$1=$ mild abnormality

$2=$ moderate abnormality

$3=$ severe abnormality

For scoring of appearance, joint swelling was categorised as grade 1 , malalignment as grade 2 and deformity as grade 3 . For movement, grade 3 was defined as the movement being impossible or only achieved with severe difficulty.

Functional indices

The Steinbrocker, HAQ and Barthel functional indices were collated independently by an occupational therapist (ED). The original Steinbrocker version was employed. ${ }^{6}$

\section{Statistics}

Correlations between the GALS score and the functional indices were tested by the Spearman correlation coefficient. Interobserver variation was assessed by Spearman correlation and Kappa statistics. ${ }^{17}$

\section{Results}

GALS locomotor screen

Responses to screening questions showed that of 83 patients, 81 complained of pain or stiffness, 53 had difficulty in dressing, and 69 had difficulty in climbing 5 stairs. The two patients not picked up by these questions both had rheumatoid arthritis in remission. Patients admitting difficulty with dressing or stairs had mean HAQ scores of $2 \cdot 3$ and $2 \cdot 1$ respectively, compared with mean HAQ scores of 0.9 or 0.6 for those without such difficulties.

Mean GALS and HAQ scores were higher for rheumatoid arthritis $(10.7$ and 2.3 respectively) compared with the miscellaneous rheumatic conditions $(5 \cdot 1$ and $1 \cdot 3)$.

The distribution of GALS scores (fig 1) is continuous but skewed with a median of 7 (range 0 to 21 ). In this study group the full range was employed, as one patient had severe deforming RA with spinal involvement. By contrast HAQ scores (fig 2) showed a bimodal distribution with a large peak at $2 \cdot 8-3 \cdot 0$.

\section{Correlation of GALS with functional indices}

Table 1 shows Spearman $r$ values for GALS scores correlated with HAQ, Barthel and Steinbrocker that range between 0.62 to 0.71 . Despite the reduced patient numbers when the single diagnosis of RA is considered, correlation remains $>0.65$ with a similar significance value. Correlation is less good for the miscellaneous rheumatic conditions excluding RA, but is still 0.46 for the HAQ. It should be remembered that for non-parametric data, Spearman correlation is preferred, which depends on rank order rather than on the absolute values. A scatterplot of GALS against HAQ is shown for the RA group and the miscellaneous group (fig 3 ). Two outlying points warrant further description. A patient with polymyalgia rheumatica (PMR) had a GALS score of 4 and HAQ of $2 \cdot 9$, and an adult well adapted to his juvenile onset arthritis (JCA) had a GALS of 11 , and a HAQ of only $0 \cdot 2$, despite flexion deformities of the knees.

When GALS is broken down into the individual components (table 2), correlation is less for the spine $(r=0.34-0.42)$ than for gait, arms or legs $(0 \cdot 49-0 \cdot 64)$. Comparing the relative value of assessment of appearance as opposed to movement, the latter correlates better with HAQ ( $r=0.69$ vs 0.54$)$.

\section{Interobserver variation}

Interobserver variability was assessed in 30 patients and gave a Spearman correlation of 0.90 . Observers agreed on the score for the individual components of GALS in $70 \%$ of cases or more. The Kappa statistics are shown in table 3, and were in the moderate to good range: significance levels were all $\mathrm{p}<0.001$, except for spine appearance, $p<0.005$. The full range of scores was used for all components except spine-appearance.

\section{Discussion}

The ideal screening test should fulfil three requirements: simplicity, reliability and validity. Let us consider each of these in turn. Certainly the GALS locomotor screen is simple and quick to perform. It takes only approximately three minutes once the patient is undressed. Results are also reliable between different observers, when a basic scoring system is employed. Agreement by kappa statistics 


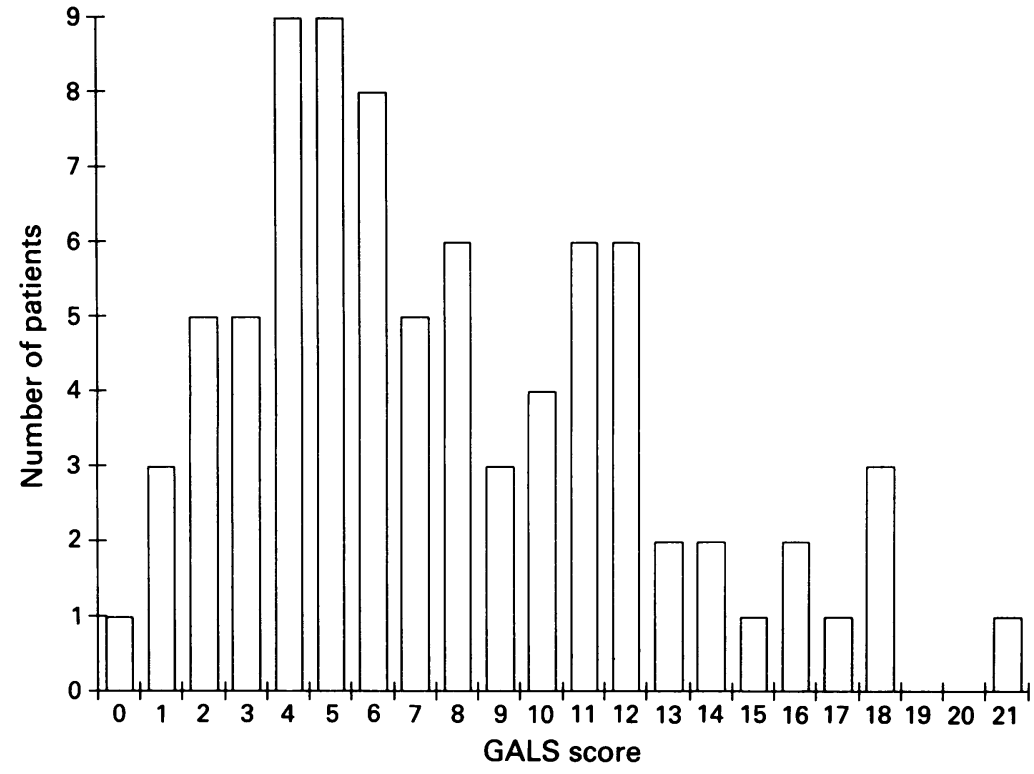

Figure 1 Histogram to show distribution of GALS scores.

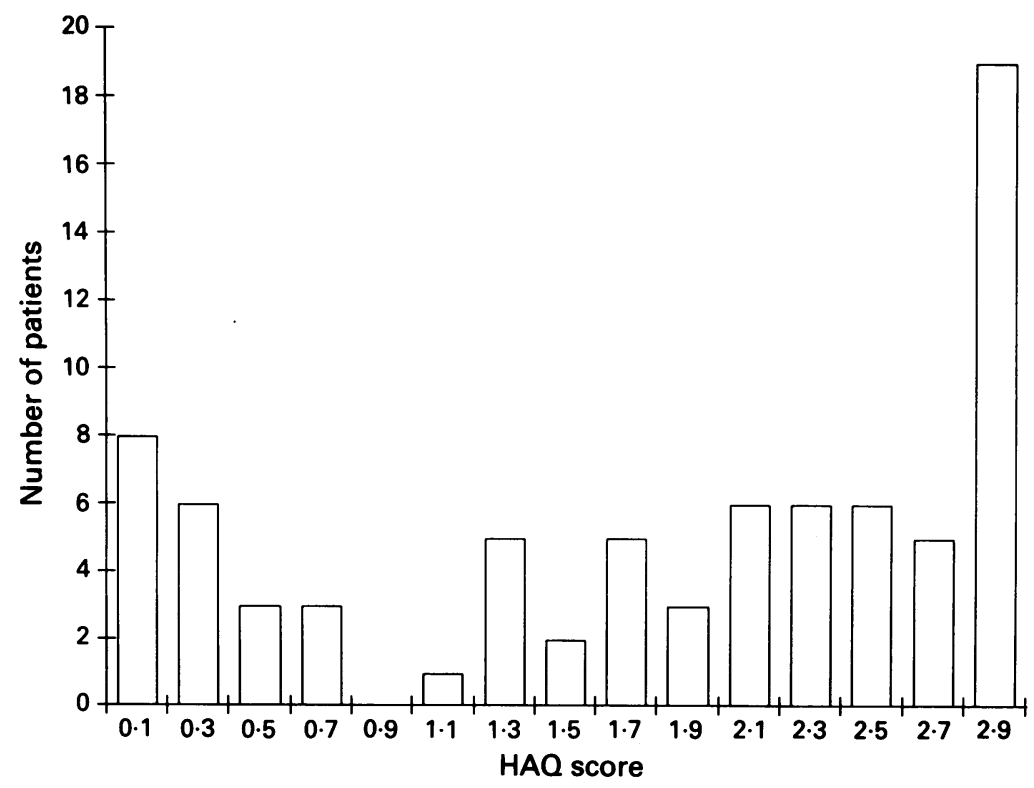

Figure 2 Histogram to show distribution of HAQ scores.

Table 1 Spearman correlation matrix of GALS v functional indices (HAQ, Barthel, Steinbrocker)

\begin{tabular}{lllll}
\hline & $H A Q$ & Barthel & Steinbrocker & \\
\hline $\begin{array}{l}\text { GALS } \\
\text { all patients }\end{array}$ & 0.71 & 0.62 & 0.65 & $\mathrm{p}<0.001$ \\
$\mathrm{n}=83$ & & 0.70 & 0.65 & $\mathrm{p}<0.001$ \\
$\begin{array}{c}\text { GALS RA only } \\
\mathbf{n}=41\end{array}$ & 0.69 & 0.31 & 0.39 & \\
$\begin{array}{c}\text { GALS non-RA } \\
\mathrm{n}=42\end{array}$ & $\begin{array}{l}0.46 \\
\mathrm{p}<0.01\end{array}$ & $\begin{array}{l}0.3<0.05 \\
\mathrm{p}<0.01\end{array}$ & \\
\hline
\end{tabular}

according to the guidelines suggested by Brennan and Silman ${ }^{18}$ was in the moderate to good range for the seven components of GALS. Intra-observer variability could not be ascertained as test-retest studies would be confounded by changes in the patient's condition over time.

This analysis, however, emphasised that scoring the appearance of the spine is most prone to variation, and that the full range of scores tends not to be used. Similarly, in developing their minimal rheumatological screen, Jones et al found poorest agreement for lateral cervical flexion. ${ }^{5}$ Spinal abnormalities tend to be more subtle than those in the limbs, and age-related changes in spinal flexibility in older patients make differentiation of normal from abnormal more difficult. The spine also correlated least well with the functional indices, although the latter tends to overrepresent limb function.

Approximately two thirds of patients responded positively to all the screening questions, and $98 \%$ had pain or stiffness. This high proportion reflects the hospital population. The questions about dressing and climbing stairs are sensitive as the HAQ scores were much higher in patients admitting problems with these activities.

The third requirement of validity is more difficult as there is no gold standard for measurement of function. New measures tend to be validated by comparison with already accepted methods. Hence, the significant correlation of the GALS screen with the Steinbrocker, Barthel and HAQ methods is reassuring. Moreover, the GALS screen is conceptually different in that it assesses physical abnormality and tests performance of specific and functionally important movements instead of relying on the patient's responses to questions which may be open to misinterpretation. Of the three indices, the HAQ has been most thoroughly investigated. It correlates well with other measures of health status and disability, including AIMS, ${ }^{19}$ Mallya and Mace index of disease activity in $\mathrm{RA},{ }^{20}$ and Functional Status Index. ${ }^{21}$ The HAQ has been evaluated in RA and osteoarthritis, although not in other musculoskeletal disorders. It has been considered the 'best buy' currently available by one author, ${ }^{22}$ and for this reason was included in this study.

In conclusion, the GALS screen does appear to be a reliable and valid indicator of functional ability.

Analysis of the individual components of GALS shows that correlation with the three functional indices is similar for gait, arms and legs but less good for the spine. In general, assessment of movement was a better predictor of function than was appearance. Although this difference was not large, it was consistent for all three indices, and reinforces the impression that an impairment which is obvious visually, may not necessarily handicap the patient. It has also been suggested that the HAQ is more influenced by upper limb function. This is supported by the better correlation of HAQ with GALS-arms $(r=0.64)$ than GALS-legs $(r=0.53)$, but the small difference suggests that the HAQ is not excessively biased.

The bimodal distribution of the HAQ scores is striking and more marked than in other studies. ${ }^{9}$ This partially represents the high proportion of inpatients in our study, who tend to be more disabled than outpatients. However, two aspects of the HAQ contribute to this and are highlighted by this study. The HAQ tends to maximise abnormality by first counting the worst of a group of responses for 


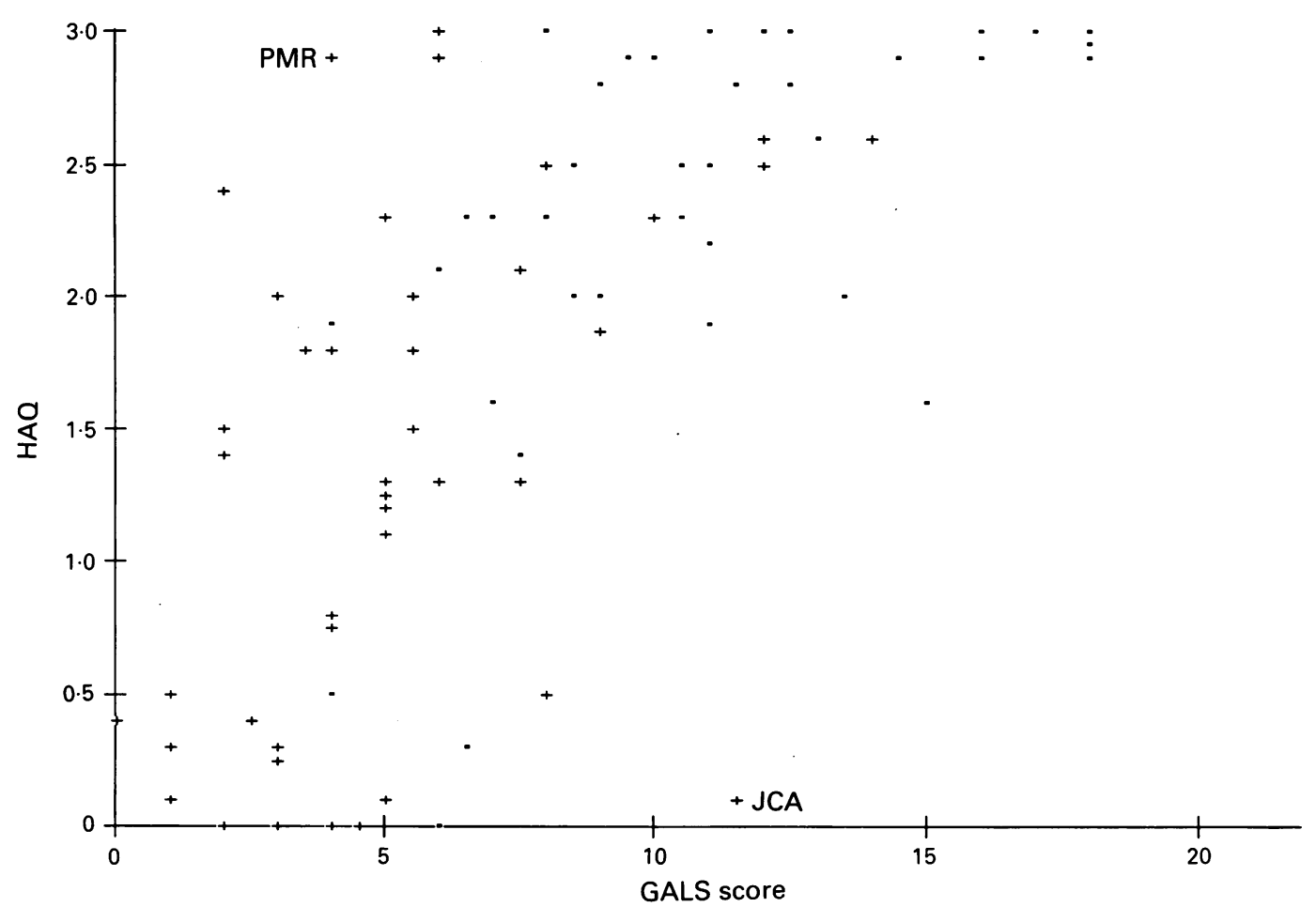

Figure 3 Scatterplot of GALS against $H A Q$ scores for $R A$ patients $(\bullet)$ and non-RA patients ( + )

Spearman correlation coefficient, $r=0 \cdot 71$

$\mathcal{F} C A=$ patient with juvenile onset chronic arthritis.

$P M R=$ patient with polymyalgia rheumatica.

Table 2 Spearman correlation of individual GALS components with functional indices

\begin{tabular}{lrll}
\hline & \multicolumn{1}{c}{$H A Q$} & Barthel & Steinbrocker \\
\hline Gait & $\mathrm{r}=0.58$ & 0.58 & 0.56 \\
Arms & 0.64 & 0.55 & 0.55 \\
Legs & 0.53 & 0.50 & 0.49 \\
Spine & 0.42 & 0.34 & 0.42 \\
Total GALS & & & \\
$\quad$ appearance & 0.54 & & \\
Total GALS & & & \\
movement + gait & 0.69 & & \\
\hline
\end{tabular}

Table 3 Interobserver variability for components of GALS. Analysis by kappa statistics

\begin{tabular}{lll}
\hline & $\begin{array}{l}\text { Observed } \\
\text { proportional } \\
\text { agreement }\end{array}$ & Kappa \\
\hline Gait & 0.73 & 0.62 \\
Arms appear & 0.83 & 0.74 \\
Arms move & 0.77 & 0.63 \\
Legs appear & 0.70 & 0.53 \\
Legs move & 0.73 & 0.52 \\
Spine appear & 0.73 & 0.49 \\
Spine move & 0.70 & 0.51 \\
\hline
\end{tabular}

a particular activity, and secondly by penalising the use of aids or adaptations. This is not necessarily a weakness of the HAQ, but it should be recognised by investigators.

Looking further into the performance of the GALS screen, the correlation between GALS and the three functional indices holds across the spectrum of rheumatic disease, but is greater when rheumatoid arthritis alone is considered. This is not surprising, as any index would perform better in a single selected population, which reduces the degree of variation. For instance, polymyalgia rheumatica and fibromyalgia are underscored by GALS relative to function-other exceptions may exist, but will still register on the screening questions. This study was based on an adult hospital population and therefore further studies are needed before the findings can be extrapolated for use in children or the general population.

GALS is only a screening test, and is not a substitute for proper locomotor clinical skills. Demonstration of an abnormality on the GALS screen should be followed up by further examination, investigation or specialist referral, to determine the correct diagnosis and management. It is, however, a useful tool to highlight facets of history and examination which are important for function, and should improve recognition of musculo-skeletal disease. The results of this study lend support to the proposition that the GALS screen should be employed in undergraduate education. ${ }^{5}$ As GALS is readily assimilated by medical students, ${ }^{4}$ it could provide a 'hatstand' of basic musculoskeletal assessment on which to 'hang' more detailed methods of examining the regional locomotor systems. It could also provide an aide-memoire for assessing disability not only in rheumatology but across the medical specialties.

1 Doherty M, Abawi J, Pattrick M. Audit of medical inpatient examination-a cry from the joint. $\mathcal{f}$ Roy Coll Phys, London 1990; 24: 115-8.

2 Morbidity statistics from general practice 1981-82. Third National Study. London: HMSO, Series MB5-1.

3 Spencer M A, Dixon A S. Rheumatological features of patients admitted as emergencies to acute general medical wards. Rheumatol Rehabil 1981; 20: 71-73.

4 Doherty M, Dacre J, Dieppe P, Snaith M. The 'GALS' locomotor screen. Ann Rheum Dis 1992; 51: 1165-69.

5 Jones A, Ledingham J, Regan M, Doherty M. A proposed minimal rheumatological screening history and examination. $\mathcal{F}$ Roy Coll Phys London, 1991; 25(2): 111-15.

6 Steinbrocker O, Traeger C H, Batterman R C. Therapeutic criteria in rheumatoid arthritis. $\mathscr{f} A M A$ 1949; 140: 659-62. 
7 Hochberg M C, Chang R W, Dwosh I, Lindsey S, Pincus $\mathrm{T}$, Wolfe $\mathrm{F}$. The American College of Rheumatology 1991 Revised Criteria for the Classification of Global Functional Status in Rheumatoid Arthritis. Arth Rheum 1992; 35: 498-502.

8 Fries J F, Spitz P, Kraines R G, Holman H. Measurement of patient outcome in arthritis. Arth Rheum 1980; 23: of patien $137-145$.

9 Kirwan J R, Reeback J S. Stanford HAQ modified to assess disability in British patients with rheumatoid arthritis. $B$ f Rheum 1986; 25: 206-9.

10 Bombardier C, Ware J, Russell I J, Larson M, Chalmers A Read J L. Auranofin therapy and quality of life in patients with rheumatoid arthritis: results of a multicenter trial. Am F Med 1986; 81: 565-78.

11 Lorig K, Lubeck D, Kraines R J, Seleznick M, Holman H $\mathrm{R}$. Outcomes of self-help education for patients with arthritis. Arthritis Rheum 1985; 28: 680-85.

12 Wolfe F, Kleinheksel S M, Cathey M A, Hawley D J, Spit $P$ W, Fries J F. The clinical value of the Stanford Health Assessment Questionnaire functional disability index in patients with rheumatoid arthritis. $\mathcal{F}$ Rheumatol 1988 ; 15 patients with $1480-88$.

13 Yelin E, Henke C, Epstein W. The work dynamics of the person with rheumatoid arthritis. Arthritis Rheum 1987; 30: $507-12$.

14 Colin C, Wade D T, Davis S, Horne V. The Barthel ADL
Index: a reliability study. International Disability Studies 1988; 10: 61-63.

15 Wade D T, Colin C. The Barthel ADL Index: a standard measure of physical disability? International Disability Studies 1988; 10: 64-7.

16 Wylie C M. Gauging the response of stroke patients to rehabilitation. Fournal of the American Geriatrics Society, 1967; 15: 797-805.

17 Cohen J A A coefficient of agreement for nominal scales. Educational and psychological measurement 1966;20: Education.

18 Brennan P, Silman A. Statistical methods for assessing observer variability in clinical measures. BMF 1992; 304: 1491-94

19 Brown J H, Kazis L E, Spitz P W, Gertman P, Fries J F, Meenan R F. The dimensions of health outcomes: a crossvalidated examination of health status measurement. $A m$ f Public Health 1984; 74: 159-61.

20 Fitzpatrick R, Newman S, Lamb R, Shipley M. A comparison of measures of health status in rheumatoid arthritis. Brit f Rheumatol 1989; 28: 201-6.

21 Liang $M$ H, Larson M G, Cullen K E, Schwartz J A Comparative measurement efficiency and sensitivity of fomparative measurement efficiency and sensitivity of Arthritis Rheum 1985; 28: 542-7.
Are health status instruments

22 Thompson P W. Functional outcome in rheumatoid arthritis. Brit $\mathcal{F}$ Rheumatol 1988; 27 (suppl 1): 37-43.

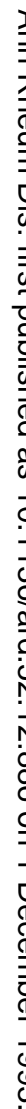

